\title{
ESTUDIO TEÓRICO DE LA ISOMERIZACIÓN DEL ÁCIDO MALEICO EN ÁCIDO FUMÁRICO: UN ENFOQUE BASADO EN EL CONCEPTO DE SUPERFICIE DE ENERGÍA POTENCIAL
}

\author{
Ricardo Ugarte \\ Instituto de Química. Facultad de Ciencias, Universidad Austral de Chile, Casilla 567, Valdivia, Chile \\ Guillermo Salgado* \\ Universidade Andrés Bello, Sede Concepción, Chile \\ Rolando Martinez \\ Universidad Andres Bello, Viña del Mar, Chile \\ José Navarrete \\ Educom, Santiago, Chile
}

Recebido em 19/3/09; aceito em 15/9/09; publicado na web em 22/2/10

\begin{abstract}
THEORETICAL STUDY OF THE ISOMERIZATION OF MALEIC ACID INTO FUMARIC ACID: AN APPROACH BASED ON THE CONCEPT OF POTENTIAL ENERGY SURFACE. A comparative study based on potential energy surfaces (PES) of 2-butanedioic and hypothetic 2-butanedioic/ $\mathrm{HCl}$ acids is useful for understanding the maleic acid isomerization. The PES enables locating conformers of minimum energy, intermediates of reactions and transition states. From contour diagrams, a set of possible reaction paths are depicted interconnecting the proposed structures. The study was carried out in absentia and in the presence of the catalyst $(\mathrm{HCl})$, using an solvatation model provided by the Gaussian software package. Clearly, the effect of $\mathrm{HCl}$ is given by new reaction paths with lower energetic barriers in relation to the reaction without catalyzing.
\end{abstract}

Keywords: potencial energy surface; isomerization; maleic acid.

\section{INTRODUCCIÓN}

Una clase particular de isómeros geométricos (cis-trans) son aquellos compuestos que difieren en el ordenamiento espacial de los grupos ligados a un enlace doble. En estos isómeros, los dos grupos unidos al átomo de carbono de un enlace doble son diferentes entre sí; lo mismo es válido para el otro átomo de carbono; sin esta condición, los dos isómeros serían indistinguibles. Normalmente, existirá una rotación libre y una restringida alrededor de un enlace simple y uno doble, respectivamente. Así, los grupos que se unen a átomos de carbono de un enlace doble permanecerán fijos a uno u otro lado del enlace. Al presentar propiedades diferentes, los isómeros cis-trans pueden ser distinguidos uno del otro.

La proporción en que coexisten en equilibrio, a una determinada temperatura, las diferentes poblaciones de isómeros depende de sus diferencias en energías libres, $\Delta \mathrm{G}^{\circ}$. No obstante, el equilibrio de interconversión se producirá siempre y cuando la barrera rotacional, representada por la energía libre de activación $\left(\Delta \mathrm{G}^{\ddagger}\right)$, no sea demasiado alta.

Un ejemplo clásico de isomería cis-trans es la representada por el ácido 2-butenodioico, que en condiciones normales es un sólido. En este compuesto el isómero cis-2-butenodioico recibe el nombre de ácido maleico, mientras que el trans-2-butenodioico se denomina ácido fumárico.

El ácido fumárico se distribuye ampliamente en la naturaleza. Se encuentra, por ejemplo, en plantas del género fumaria (Fumaria officinalis), en el liquén Cetraria islandica y en hongos de las especies Boletus y Agaricus. ${ }^{1}$ La forma ionizada del ácido fumárico (fumarato) es un intermediario clave en el ciclo de Krebs. El ácido maleico es un producto de síntesis obtenido a partir de la hidrólisis del anhídrido maleico; este último, se produce a partir de la oxidación catalítica $\left(\mathrm{V}_{2} \mathrm{O}_{5}\right.$, aire, $\left.400-500{ }^{\circ} \mathrm{C}\right)$ del benceno.

De acuerdo a los valores del calor de formación de ambos isómeros en fase sólida $(\text { Tabla } 1)^{2}$ se deduce que el isómero cis es $\approx 4.9 \mathrm{kcal}$

*e-mail: gsalgado41@gmail.com $\mathrm{mol}^{-1}$ menos estable que el isómero trans. Esta diferencia se explica sí consideramos los posibles puentes de hidrógeno intermoleculares en ambos isómeros (dos para la forma trans y uno para la forma cis) y la proximidad de los dos grupos carbóxilo en el isómero cis; si bien la repulsión estérica que implica esta proximidad resulta minimizada por la formación de un puente de hidrógeno intramolecular entre el grupo $\mathrm{OH}$ de un carbono y el oxígeno carbonilo de otro, estabilizándose así la estructura cis.

Tabla 1. Propiedades físicas del ácido 2-butenodioico en estado sólido

\begin{tabular}{|c|c|c|}
\hline Isómero geométrico & Cis & Trans \\
\hline Fórmula & $\mathrm{C}_{4} \mathrm{H}_{4} \mathrm{O}_{4}$ & $\mathrm{C}_{4} \mathrm{H}_{4} \mathrm{O}_{4}$ \\
\hline Punto de fusión $/{ }^{\circ} \mathrm{C}^{\mathrm{a}}$ & $138-139$ & 287 \\
\hline$\Delta \mathrm{H}^{\circ}$ de formación $/ \mathrm{kcal} \mathrm{mol}^{-1}$ & $-188,95$ & $-193,84$ \\
\hline $\mathrm{S} / \mathrm{cal} \mathrm{grado}^{-1} \mathrm{~mol}^{-1}$ & 38,1 & 39,7 \\
\hline Calor de sublimación/kcal mol${ }^{-1}$ & 25,19 & 29,54 \\
\hline
\end{tabular}

a (cristalizado desde agua)

El ácido fumárico puede ser obtenido por isomerización del ácido maleico colocado en un tubo sellado a una temperatura de $200{ }^{\circ} \mathrm{C} ;{ }^{3}$ calentando una disolución acuosa de ácido maleico en un tubo sellado sobre $130{ }^{\circ} \mathrm{C} ;{ }^{4}$ a partir de una disolución de ácido maleico calentada aproximadamente a $100{ }^{\circ} \mathrm{C}$ con cualesquiera de los siguientes catalizadores, $\mathrm{HBr}, \mathrm{HCl}, \mathrm{KSCN} ;^{5-7}$ irradiando con luz ultravioleta una disolución de ácido maleico que contenga una gota de bromo. ${ }^{1}$ En las reacciones de isomerización en disolución acuosa, el ácido fumárico cristaliza a medida que va siendo formado, desplazando el equilibrio de reacción y dando como resultado un alto rendimiento en producto. Por otro lado, la luz ultravioleta provoca que en una disolución de ácido fumárico se establezca un equilibrio entre los dos isómeros.

Una explicación satisfactoria de la interconversión cis-trans debería implicar un mecanismo mediante el cual el enlace doble $\mathrm{C}=\mathrm{C}$ se debilite al punto de asemejarse a un enlace simple. Así, sería 
posible la rotación a través de este enlace. Los mecanismos que han sido descritos son los siguientes:

a) Térmico: La temperatura modula la razón cis/trans e influye en la velocidad de isomerización debido, entre otras cosas, al efecto que provoca en la amplitud de vibración del doble enlace.

b) Catalítico: La isomerización de ácido maleico a ácido fumárico catalizada por ácidos y sales ha sido estudiada experimentalmente, ${ }^{5,8}$ sugiriéndose el siguiente mecanismo para la catálisis ácida (Figura 1a): ${ }^{8}$ La etapa (a) involucra la adición de un protón al oxígeno carbonilo del carbono 1, la adición de un anión $\mathrm{X}$ al carbono 3, y el desplazamiento del doble enlace entre los carbonos 2 y 3 hacia los carbonos 1 y 2. Esta etapa se considera el paso determinante de la velocidad; en cambio las etapas (b) y (c) se consideran rápidas. Aunque la etapa (a) se formula como una reacción termolecular, es probable que la adición del protón preceda a la del anión. Debido a que el anión participa en la etapa determinante de la velocidad, su naturaleza debería afectar la velocidad de reacción.

Otro mecanismo que en alguna oportunidad fue propuesto se refiere a la adición del protón o de HX al doble enlace, lo cual es bastante común en olefinas; ${ }^{9}$ sin embargo, la evidencia experimental refutó este mecanismo. ${ }^{10,11}$

c) Fotocatalítico: La isomerización fotoquímica del ácido maleico es producida por bromo en presencia de luz ultravioleta. ${ }^{1} \mathrm{El} \mathrm{radical} \mathrm{libre}$ que resulta del ataque de un átomo de bromo, sobre el carbono del doble enlace, puede sufrir una rotación alrededor de su enlace C-C central.

No obstante, el avance que se ha logrado en la comprensión del proceso de interconversión del ácido maleico en ácido fumárico, existe alguna controversia en los valores citados de las energías de activación y $\Delta \mathrm{H}^{\circ}$ de isomerización. Además, dependiendo de las condiciones de experimentación en disolución acuosa, ocurren reacciones colaterales que afectan la cinética observada del proceso, tales como, la generación de ácido málico, ${ }^{12} \mathrm{o}$ ácido halosuccinico en la reacción catalizada por $\mathrm{HCl},{ }^{8}$ y presumiblemente ácido dibromosuccinico o ácido bromofumárico en la reacción catalizada por átomos de bromo. ${ }^{13}$

En consecuencia, se realizó un estudio teórico que aporte alguna comprensión del fenómeno en cuestión, aislado de las complejidades inherentes a la experimentación. Así, el objetivo del presente trabajo consiste en un análisis de la reacción de isomerización del ácido maleico, basado en la superficie de energía potencial o mapa conformacional de la molécula. En particular, se hizo un estudio comparativo entre la isomerización no catalizada y catalizada, el cual se reduce a explicar cualitativamente el efecto del ácido clorhídrico en la interconversión cis-trans; con este propósito, se exploró el espacio conformacional del ácido 2-butenodioico y de la especie hipotética que resulta de la adición de ácido clorhídrico, obteniéndose así, información acerca de posibles estados de transición, intermediarios de reacción y barreras de energía impuestas a la rotación a través del doble enlace.

\section{MÉTODO DE CÁLCULO}

Los cálculos teóricos fueron realizados en fase acuosa utilizando el modelo de solvatación IEFPCM ${ }^{14}$ del programa computacional Gaussian, ${ }^{15,16}$ implementado en un computador Dell PowerEdge R805. En el modelo de solvatación IEFPCM (Integral-Equation-Formalism Polarizable Continuum Model) se trata al disolvente como un medio continuo caracterizado por su constante dieléctrica, mientras que al soluto se le describe inmerso en una cavidad, de forma y dimensiones apropiadas, en el medio continuo. La cavidad define una superficie cerrada que separa al soluto del disolvente y es utilizada para formular las ecuaciones electrostáticas que caracterizan la interacción soluto-disolvente. El soluto es representado por una distribución de carga implantada en la superficie de la cavidad. La respuesta dieléctrica del disolvente a la presencia del soluto se representa a través de una carga superficial inducida, o apar-
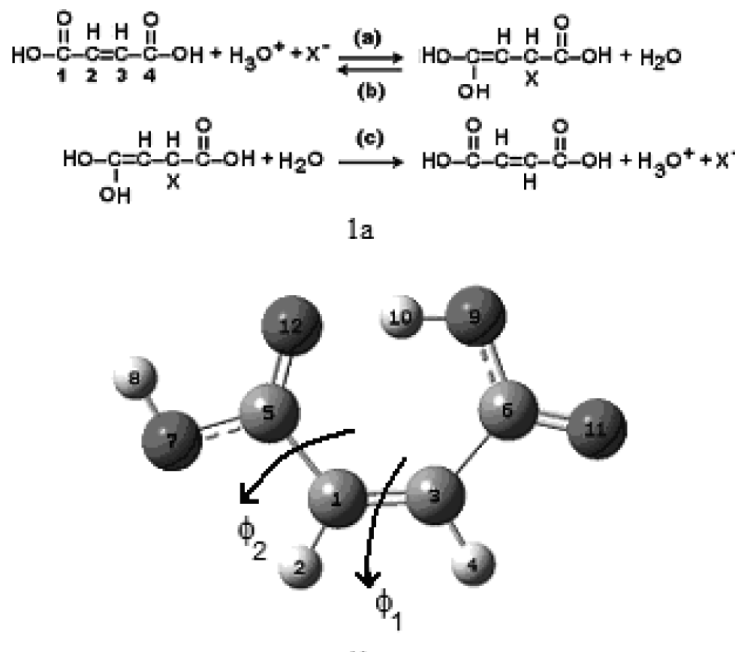

$1 \mathrm{~b}$

Figura 1. (a) Representación esquemática del mecanismo probable; (b) angulos diedros $\Phi_{1} y \Phi_{2}$. En la conformación mostrada $\Phi_{1}=0^{\circ}$ (ácido maleico); $\Phi_{2}=180^{\circ} ; \Phi_{3}=0^{\circ} . \Phi_{1}\left(C_{5}-C_{1}-C_{3}-C_{6}\right) ; \Phi_{2}\left(O_{7}-C_{5}-C_{1}-C_{3}\right) ; \Phi_{3}\left(C_{1}-C_{3}-C_{6}-O_{9}\right)$

ente, diseminada sobre la cavidad. Esta carga inducida genera un campo eléctrico o "campo de reacción", que modifica la descripción del soluto respecto a este mismo en fase gaseosa. Así, la interación soluto-disolvente modifica los estados previos (molécula aislada-disolvente no polarizado) de ambos componentes dirigiéndolos hacia un estado más estable. Este proceso de interacción es descrito a nivel mecano-cuántico por un operador hamiltoniano efectivo, construido a partir del operador hamiltoniano estándar (fase gaseosa) al cual se le adicionan términos que describen la interacción soluto-disolvente. En este modelo se utilizan funciones de Green para definir los operadores integrales que determinarán la carga superficial aparente.

El conocido formalismo $\mathrm{RHF}^{17}$ fue utilizado con la base estándar 6-31G(d). La siguiente palabra clave y opciones fue especificada en la sección ruta del programa Gaussian: $\mathrm{scrf}=(\mathrm{iefpcm}$,solvent=water).

La conformación de estos compuestos se describe mediante los ángulos diedros $\Phi_{1}$ y $\Phi_{2}$ (Figure 1b). El ángulo diedro $\Phi_{1}$ de los átomos enlazados $C_{5}-C_{1}-C_{3}-C_{6}$ es el ángulo entre los planos $C_{5}-C_{1}-C_{3} y$ $\mathrm{C}_{1}-\mathrm{C}_{3}-\mathrm{C}_{6}$. Visto desde el lado de $\mathrm{C}_{5}, \Phi_{1}$ es positivo para una rotación en sentido del reloj del extremo distal respecto al proximal y negativo en caso contrario. Si $\Phi_{1}=0^{\circ}$ la molécula presenta el ordenamiento cis-planar de los enlaces $\mathrm{C}_{5}-\mathrm{C}_{1}$ y $\mathrm{C}_{3}-\mathrm{C}_{6}$ (ácido maleico); $\operatorname{con} \Phi_{1}=180^{\circ}$ obtenemos el ordenamiento trans-planar (ácido fumárico).

El análisis conformacional se basa en la superficie de energía potencial de las moléculas (PES), la cual se construye haciendo variar los ángulos diedros $\Phi_{1}$ y $\Phi_{2}$ en incrementos de $20^{\circ}$ desde -180 a $+180^{\circ}$. A partir de una estructura inicial escogida, una optimización de la geometría se realiza en cada punto del plano $\Phi_{1} / \Phi_{2}$, manteniendo "congelados" los ángulos diedros en sus respectivos valores y dejando que el resto de la molécula optimice libremente su geometría (PES relajada). Así, se construye un gráfico que representa la energía de la molécula en función de las conformaciones definidas por los ángulos diedros. La naturaleza de los puntos estacionarios sobre la PES se verifica calculando los valores propios de la matriz Hessiana; los mínimos de energía no tienen valores propios negativos, mientras que los puntos de silla de primer orden (estructuras de transición) tienen exactamente un valor propio negativo. Es preciso realizar una optimización total de la geometría para asegurarnos de encontrar los mínimos locales, puntos de silla y el mínimo global (estructura de menor energía) correspondiente a cada molécula; para esto se escoge como estructuras de inicio un conjunto de geometrías que se ubiquen en una vecindad de los mínimos o puntos de silla encontrados 
en la PES y se procede a su optimización sin imponer restricciones. En orden a facilitar el análisis de los datos se construirán diagramas de contorno constituidos de mínimos, puntos de silla y caminos de reacción.

\section{RESULTADOS Y DISCUSIÓN}

\section{Reacción no catalizada}

La Figura 2 muestra la PES del ácido 2-butenodioico. Se observa que la barrera teórica impuesta para la rotación a través del enlace $\mathrm{C}=\mathrm{C}$, es demasiado alta para que la interconversión ocurra a temperatura ambiente. Por lo tanto, cada isómero puede existir independientemente del otro a esta temperatura.

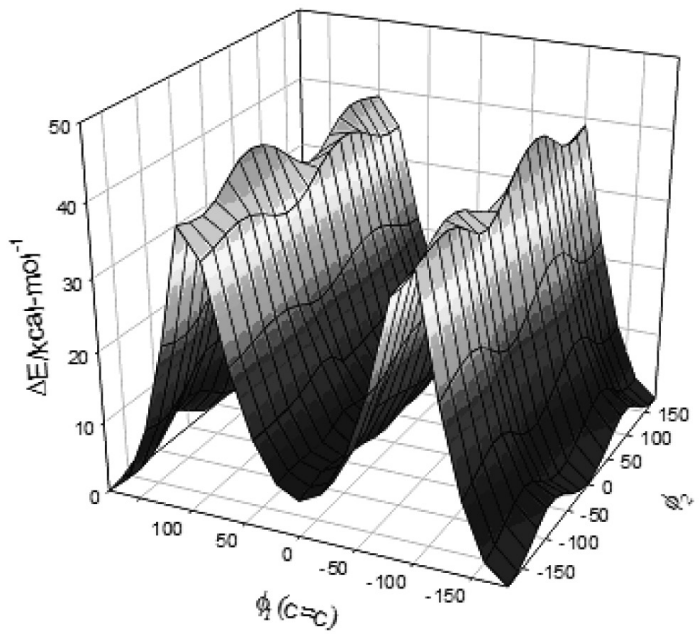

Figura 2. Superficie de energía potencial relajada del ácido 2-butenodioico. Los valores de energía relativa $(\Delta E)$ en $\mathrm{kcal} \mathrm{mol}^{-1}$ se informan en relación al mínimo global Trans $I:\left[\Phi_{1}, \Phi_{2}\right]=\left[180^{\circ}, 180^{\circ}\right]$

En el diagrama de contorno de la Figura 3 se localizan los mínimos y puntos de silla correspondientes a las conformaciones que se enumeran en la Tabla 2. El mínimo global corresponde a Trans I. Debido a consideraciones de simetría Cis I: $\left[+1.21^{\circ},+178.02^{\circ}\right]$ y Cis I': $\left[+1.21^{\circ},-87.86^{\circ}\right]$ son idénticas (lo mismo acontece con el otro par de estructuras Cis I y Cis I'). Además, Cis I: $\left[+1.21^{\circ},+178.02^{\circ}\right]$ y Cis I: $\left[-1.21^{\circ},-178.02^{\circ}\right]$ representan un par de enantiómeros, al igual que Cis I': $\left[+1.21^{\circ},-87.86^{\circ}\right]$ y Cis I': $\left[-1.21^{\circ},+87.86^{\circ}\right]$. Lo anterior, es válido para Cis II y Cis II'. La diferencia entre Cis III y Cis IV, se relaciona con un ángulo diedro $\Phi_{3}$ diferente (Cis III $=0^{\circ}$, Cis IV $=180^{\circ}$, ver Figura $1 \mathrm{~b}$ ).

De acuerdo a la Tabla 2, la diferencia en energía entre Cis III (forma adoptada por el ácido maleico en fase sólida) ${ }^{18}$ y Trans I es de $8.3 \mathrm{kcal} \mathrm{mol}^{-1}$, mientras que el valor experimental en fase sólida es $\approx 4.9 \mathrm{kcal} \mathrm{mol}^{-1}$. Nuestros resultados muestran que Cis I es más estable que Cis III; en el primero, el ángulo diedro $\Phi_{3}$ es prácticamente recto $( \pm 87.86)$, lo cual es un indicio de la no existencia del puente de hidrógeno en este confórmero. No obstante, un cálculo RHF en fase gas utilizando la base 6-31G(d), da como resultado que Cis III es más estable que Cis I. Así, el efecto del disolvente, a este nivel de teoría, se puede traducir en una "desestabilización" del puente de hidrógeno intramolecular, lo que implica un aumento en la estabilidad relativa del confórmero Cis I respecto a Cis III; en este último, es también importante la repulsión entre los oxígenos $\mathrm{O}(9)$ y $\mathrm{O}(12)$ (Figura 1b). Cierta evidencia experimental, ${ }^{19}$ proveniente de espectroscopía infrarroja, señala que en disolución acuosa el ácido maleico no presentaría un puente de hidrógeno

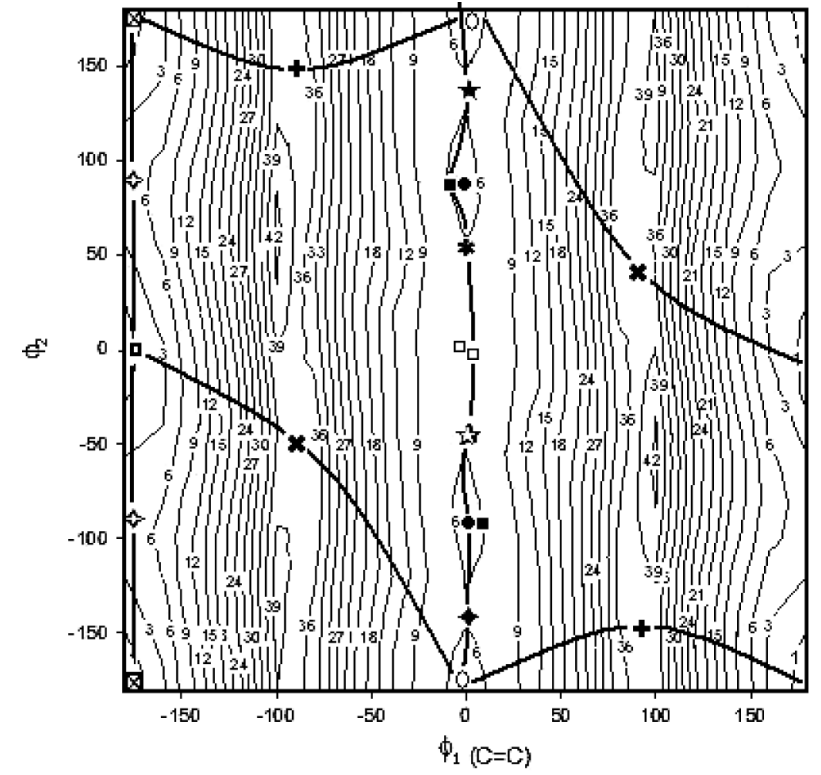

Figura 3. Diagrama de contorno de la PES relajada del ácido 2-butenodioico. En trazo más oscuro se observan algunos de los posibles caminos de

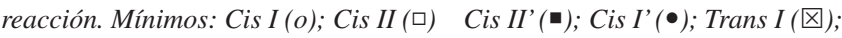
Trans II (ם). Estados de Transición: TSa $(\omega) ; \operatorname{TSb}(\zeta) ; T S b '(\varphi) ; T S c(\gamma)$; $T S d(\eta) ; T S_{1}(\Omega) ; T S_{2}([)$

Tabla 2. Angulos diedros, energías absolutas $\left(\mathrm{E}_{\mathrm{HF}}\right)$ y relativas $(\Delta \mathrm{E})$ de los mínimos locales y estados de transición de la PES del ácido 2-butenodioico. Energías relativas se informan respecto al mínimo global

\begin{tabular}{|c|c|c|c|c|}
\hline Confórmero & $\Phi_{1} /^{\circ}$ & $\Phi_{2} /^{\circ}$ & $\mathrm{E}_{\mathrm{HF}} /$ Hartree & $\Delta \mathrm{E} / \mathrm{kcal} \mathrm{mol}^{-1}$ \\
\hline Cis I & \pm 1.21 & \pm 178.02 & -453.29505281 & 5.1 \\
\hline Cis I' & \pm 1.21 & \pm 87.86 & -453.29505281 & 5.1 \\
\hline Cis II & \pm 1.36 & \pm 2.15 & -453.29358885 & 6.0 \\
\hline Cis II' & \pm 1.36 & \pm 88.08 & -453.29358885 & 6.0 \\
\hline Cis III & 0 & 180 & -453.28990280 & 8.3 \\
\hline Cis IV & 0 & 180 & -453.28807573 & 9.5 \\
\hline Trans I & 180 & 180 & -453.30317208 & 0 \\
\hline Trans II & 180 & 0 & -453.30274160 & 0.3 \\
\hline Trans III & 180 & 0 & -453.30222773 & 0.6 \\
\hline $\mathrm{TS}_{1}$ & \pm 90 & \pm 43.87 & -453.22362541 & 49.9 \\
\hline $\mathrm{TS}_{2}$ & \pm 90 & \pm 143.65 & -453.22061241 & 51.5 \\
\hline $\mathrm{TSa}$ & 0 & 50.33 & -453.28667335 & 10.4 \\
\hline $\mathrm{TSb}$ & 0 & 135.57 & -453.28790008 & 9.6 \\
\hline TSb' & 0 & -44.43 & -453.28790008 & 9.6 \\
\hline $\mathrm{TSc}$ & 0 & -139.0 & -453.28923412 & 8.8 \\
\hline TSd & 180 & \pm 89.54 & -453.29591079 & 4.6 \\
\hline
\end{tabular}

intramolecular ya que éste no es lo suficientemente fuerte como para resistir el efecto del agua que conlleva a la formación de un puente de hidrógeno intermolecular.

La energía de isomerización Cis $\rightarrow$ Trans calculada entre los dos confórmeros más estables (Cis I y Trans I) es de $-5.1 \mathrm{kcal} \mathrm{mol}^{-1}$.

A partir del calor de sublimación y $\Delta \mathrm{H}^{\circ}$ de formación de la Tabla 1 se deduce que la diferencia en energía, en fase gaseosa, de estos isómeros es de sólo $0,55 \mathrm{kcal} \mathrm{mol}^{-1}$. En fase gaseosa, en principio no existe la posibilidad de formación de puentes de hidrógeno intermolecular. Por lo tanto, esta pequeña diferencia en energía entre ambos isómeros, se explica de manera natural por el efecto estabilizador del puente de hidrógeno intramolecular en el ácido maleico. Resumiendo, tanto en fase gas ${ }^{20}$ como en fase sólida, el confórmero más estable 
del ácido maleico es el que presenta el puente de hidrógeno intramolecular (Cis III, ver Figura 1b). En disolución acuosa, lo anterior no es necesariamente cierto.

En relación a los estados de transición, se encontraron dos, TS y $\mathrm{TS}_{2}$, con sus respectivas imágenes especulares cuyas energías son lo suficientemente altas $\left(\approx 45 \mathrm{kcal} \mathrm{mol}^{-1}\right)$ como para impedir que la isomerización cis-trans ocurra a temperatura ambiente. El resto de los estados de transición ( $\mathrm{TSa} \rightarrow \mathrm{d}$ ) son menores en energía, debido a que se relacionan con una rotación alrededor de un enlace simple. En el diagrama de contorno (Figura 3) se observan algunos caminos de reacción, que involucran rotaciones alrededor de un enlace simple: Cis II $[+/-] \leftrightarrow \mathrm{TSa} \leftrightarrow$ Cis II' $[-/+] \leftrightarrow \mathrm{TSb} \leftrightarrow$ Cis I [-/-]; Cis II [+/-] $\leftrightarrow$ TSb' $\leftrightarrow$ Cis I' $[+/-] \leftrightarrow$ TSc $\leftrightarrow$ Cis I $[+/+]$ y Trans I $\leftrightarrow$ TSd $\leftrightarrow$ Trans II. Los movimientos a través de $\Phi_{2}=-180^{\circ} \rightarrow 180^{\circ} \operatorname{con} \Phi_{1} \approx$ $\left[-1.4^{\circ}: 1.4^{\circ}\right]$ involucran barreras de energía en el intervalo de 3.7-5.3

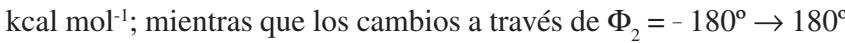
$\operatorname{con} \Phi_{1}=\left[180^{\circ}\right]$ implican barreras de $\approx 4.5 \mathrm{kcal} \mathrm{mol}^{-1}$. Lo anterior, indicaría la existencia de un equilibrio, a temperatura ambiente, entre estas distintas conformaciones.

Una teoría que intenta explicar el mecanismo cinético de esta clase de reacciones, ${ }^{21}$ clasifica a éstas, en isomerizaciones de alta (E $\left.\approx 45 \mathrm{kcal} \mathrm{mol}^{-1}\right)$ o baja $\left(\mathrm{E} \approx 25 \mathrm{kcal} \mathrm{mol}^{-1}\right)$ energía de activación. La primera procede por un estado electrónico singulete a través de todo el proceso, sin desacoplamiento de los espines de los electrones del enlace $\pi$; mientras que la segunda, a aproximadamente $\Phi_{1}=90^{\circ}$, involucra una transición a un estado triplete (biradical), pero la baja probabilidad de éste evento (isomerización no adiabática) reduce la velocidad de reacción a aproximadamente el mismo valor que el primer mecanismo. Si bien la teoría fue desarrollada en gases, se demostró que era útil para estudios en fase líquida o en disolución. Según evidencia experimental, la energía de activación para la isomerización no catalizada del ácido maleico en disolución acuosa es del orden de $21 \mathrm{kcal} \mathrm{mol}^{-1}{ }^{12}$ Nuestros cálculos respecto a la superficie de energía potencial se realizaron asumiendo estado singulete de la molécula, obteniéndose un valor de aproximadamente $45 \mathrm{kcal} \mathrm{mol}^{-1}$, en contradicción con el valor reportado experimentalmente. Por lo tanto, es probable que la reacción proceda por el mecanismo que involucra a la especie biradicalaria.

\section{Reacción catalizada}

Para dar cuenta del efecto catalítico del ácido clorhídrico en la reacción de isomerización se construyó la PES (Figura 4) de una hipotética molécula que puede representar complejos activados o intermediarios de reacción. Esta molécula, que teóricamente simplifica bastante el problema, se forma a partir del ácido 2-butenodioico adicionando un protón al oxígeno del grupo carbonilo y un ión cloruro al carbono del doble enlace (Figura 1a). La supuesta molécula explica ciertos hechos experimentales ${ }^{7}$ y fue postulada en un mecanismo para la isomerización catalizada por ácidos. ${ }^{8}$ Consideramos que la hipótesis simplificadora retiene los rasgos principales de la reacción. Es notable la diferencia entre ambas PES; en esta última se aprecian caminos "cuesta abajo" que van desde $\Phi_{1} \approx 0$ (Cis) hasta $\Phi_{1} \approx 150$ ( $\approx$ Trans), en toda la extensión del ángulo diedro $\Phi_{2}$.

En el diagrama de contorno de la Figura 5 se localizan algunos posibles estados de transición e intermediarios de reacción; además, se dibuja un par de probables caminos de reacción que interconecta a las especies moleculares postuladas. En la Tabla 3 se resumen los cálculos. Debido a que estamos interesados en diferencias de energía, los cálculos para los reactantes $(\mathrm{Cis} \mathrm{I}+\mathrm{HCl}$ ) y los productos (Trans I $+\mathrm{HCl}$ ) no consideran el $\mathrm{HCl}$ disociado en medio acuoso. Por ejemplo, la energía de isomerización de $(\mathrm{Cis} \mathrm{I}+\mathrm{HCl}) \rightarrow($ Trans $\mathrm{I}+\mathrm{HCl})$, es la misma que $\left(\right.$ Cis $\left.\mathrm{I}+\mathrm{H}_{3} \mathrm{O}^{+}+\mathrm{Cl}^{-}\right) \rightarrow\left(\right.$ Trans $\left.\mathrm{I}+\mathrm{H}_{3} \mathrm{O}^{+}+\mathrm{Cl}^{-}\right)$.

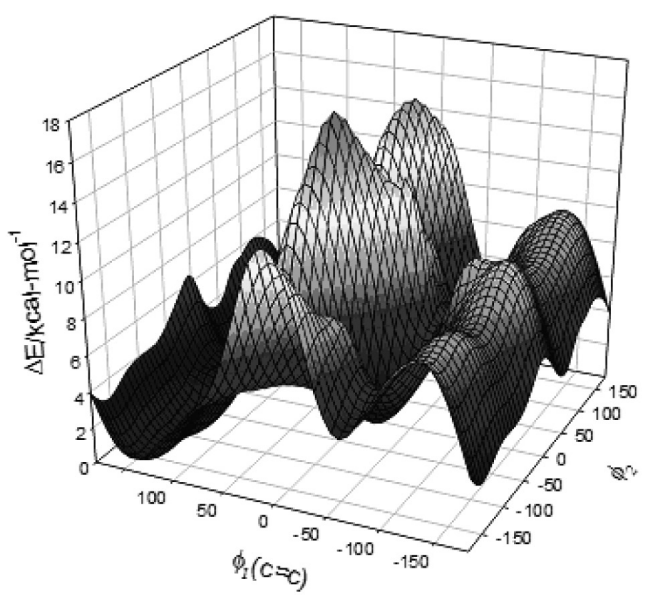

Figura 4. PES relajada del hipotético ácido 2-butenodioico/HCl. Los valores de energía relativa $(\Delta E)$ en $\mathrm{kcal} \mathrm{mol}^{-1}$ se informan en relación al mínimo global no optimizado: $\left[\Phi_{l}, \Phi_{2}\right]=\left[140^{\circ}, 60^{\circ}\right]$

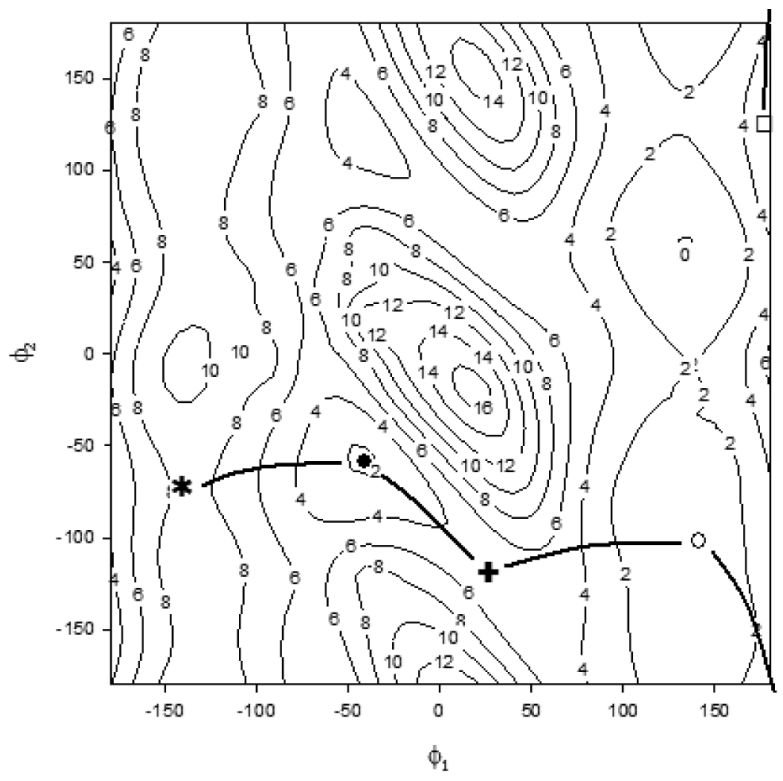

Figura 5. Diagrama de contorno de la PES relajada del hipotético ácido 2-butenodioico/HCl. En trazo más oscuro se observan dos posibles coordenadas de reacción: $T S_{I} \rightarrow I a \rightarrow T S_{I I}$ y $T S_{I} \rightarrow I b \rightarrow T S_{I I I} T S_{I}\left([) ; I a(\bullet) ; T S_{I I}\right.$ ( $)$; $I b(o) ; T S_{I I I}(9)$

Tabla 3. Angulos diedros, energías absolutas $\left(\mathrm{E}_{\mathrm{HF}}\right)$ y relativas $(\Delta \mathrm{E})$ del ácido 2-butenodioico/HCl. Energías relativas se informan respecto a los reactantes $(\mathrm{Cis} \mathrm{I}+\mathrm{HCl})$

\begin{tabular}{lcccc}
\hline Confórmero & $\Phi_{1} /{ }^{\circ}$ & $\Phi_{2} /{ }^{\circ}$ & $\mathrm{E}_{\mathrm{HF}} /$ Hartree & $\Delta \mathrm{E} / \mathrm{kcal} \mathrm{mol}^{-1}$ \\
\hline $\mathrm{TS}_{\mathrm{I}}$ & 26.37 & -115.63 & -913.33336395 & 19.3 \\
$\mathrm{TS}_{\mathrm{II}}$ & -138.08 & -74.87 & $-913,32745237$ & 23.0 \\
$\mathrm{TS}_{\mathrm{III}}$ & 180 & 122.68 & -913.33117051 & 20.6 \\
$\mathrm{Ia}$ & -39.99 & -60.01 & -913.33776144 & 16.5 \\
$\mathrm{Ib}$ & 137.53 & -98.32 & -913.34050438 & 14.8 \\
Cis I + HCl & & & -913.36407290 & 0 \\
Trans I + HCl & & & -913.37219210 & -5.1 \\
\hline
\end{tabular}

La diferencia de energía entre los reactantes $(\mathrm{Cis} \mathrm{I}+\mathrm{HCl}$ ) y el complejo activado $\mathrm{TS}_{\mathrm{I}}$ es de 19,3 $\mathrm{kcal} \mathrm{mol}^{-1}$, bastante menor que el valor calculado teóricamente para la reacción no catalizada, y algo mayor que el reportado experimentalmente para la reacción cata- 
lizada (18.4 $\left.\mathrm{kcal} \mathrm{mol}^{-1}\right) .{ }^{12}$ El proceso a través de la coordenada de reacción (Figura 6) involucra, en general, un movimiento acoplado de ambos diedros.

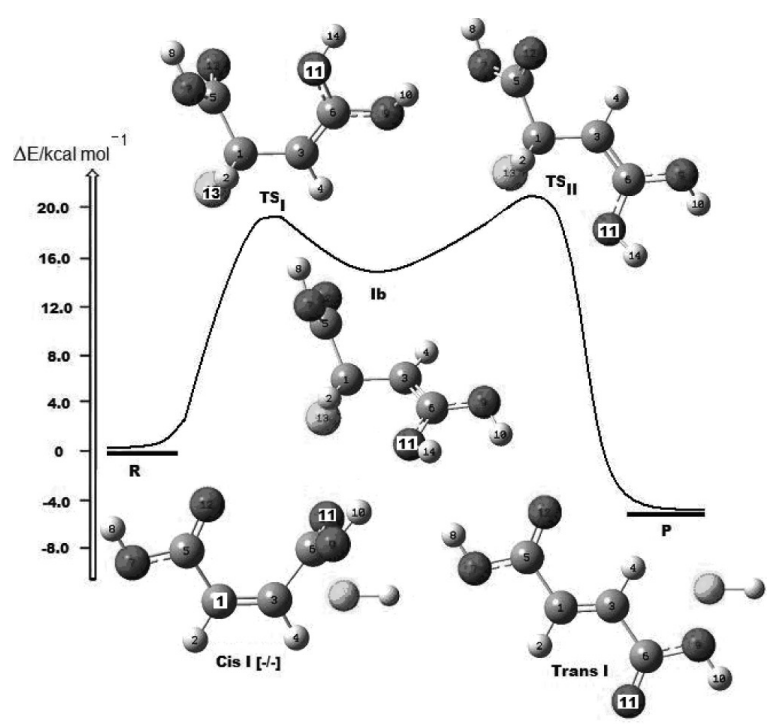

Figura 6. Esquema de la coordenada de reacción para la reacción catalizada $\Phi_{1}:\left[C_{5}-C_{1}-C_{3}-C_{6}\right] ; \Phi_{2}:\left[O_{7}-C_{5} C_{1}-C_{3}\right]$

En la reacción catalizada con $\mathrm{HCl}$ se postula que al momento de unirse el protón a $\mathrm{O}(11)$ en el confórmero Cis I, el anión ataca al carbono C(1) sp2 generándose un carbono sp3; esta perturbación de la geometría provoca movimientos de los diedros $\Phi_{1}, \Phi_{2}$ y $\Phi_{3}$, dando como resultado $\mathrm{TS}_{\mathrm{I}}$. A continuación, una rotación bastante pronunciada del diedro $\Phi_{1}$, provoca un alejamiento del $\mathrm{Cl}(13)$ y el $\mathrm{O}(11)$; estos movimientos atómicos guían a una estructura "cuasi trans" del intermediario Ib y culmina en un movimiento acoplado de los diedros $\Phi_{1}$ y $\Phi_{2}\left(\mathrm{TS}_{\mathrm{II}}\right)$. La eliminación de $\mathrm{HCl}$ y rotación en los ángulos diedros $\Phi_{1}$ y $\Phi_{2}$ conduce finalmente al confórmero Trans I.

\section{CONCLUSIONES}

Los métodos computacionales son los métodos de elección para la determinación de muchas propiedades moleculares, más aún cuando no se disponen de datos experimentales. En el presente estudio, estos métodos se emplean para construir las superficies de energía potencial del ácido 2-butenodioico y del hipótetico ácido 2-butenodioico/ $\mathrm{HCl}$, intentando demostrar que un estudio comparativo de ambas superficies puede resultar útil en la comprensión de los mecanismos subyacentes en la reacción de isomerización del ácido maleico a ácido fumárico. En general, se demostró que el efecto del catalizador es generar nuevos caminos de reacción que posibilitan una menor energía de activación respecto a la reacción en ausencia de catalizador.

\section{AGRADECIMIENTOS}

Los autores desean expresar su agradecimientos a la Dirección de Investigación y Desarrollo de la Universidad Austral de Chile
(Proyecto: S-2008-26/DID-UACh) por su generoso apoyo en la adquisición del computador.

\section{REFERENCIAS}

1. Castro, A. J.; Ellenberger, S. R.; Sluka, J. P.; J. Chem. Educ. 1983, 60, 521.

2. Mallard, W. G.; Linstrom, P. J., eds.; NIST Chemistry WebBook, NIST Standard Reference Database, Number 69, June 2005, National Institute of Standards and Technology, Gaithersburg MD, 20899; http://webbook. nist.gov.

3. González, J. R.; Gutiérrez, M. A.; Gutiérrez, J. I.; González, M. P.; González, J. A.; Chem. Eng. Process 1991, 30, 15.

4. Kiyama, R.; Minomura, S.; The Review of Physical Chemistry of Japan 1954, $22,4$.

5. Terry, E. M.; Eichelberger, L.; J. Am. Chem. Soc. 1925, 47, 1402.

6. Seltzer, S.; J. Am. Chem. Soc. 1961, 83, 1861.

7. Meek, J. S.; J. Chem. Educ. 1975, 52, 541.

8. Nozaki, K.; Ogg, R.; J. Am. Chem. Soc. 1941, 63, 2583.

9. Price, C. C.; Meister, M.; J. Am. Chem. Soc. 1939, 61, 1595.

10. Horrex, C.; Trans. Faraday. Soc. 1937, 33, 570.

11. Larsen, J. W.; Bouis, P. A.; J. Org. Chem. 1973, 38, 1415.

12. Davies, M.; Evans, F. P.; Trans. Faraday Soc. 1956, 52, 74.

13. Derbyshire, D. H.; Waters, W. A.; Trans. Faraday Soc. 1949, 45, 749.

14. Tomasi, J.; Mennucci, B.; Cancès, E.; J. Mol. Struct. (Theochem). 1999, $464,211$.

15. Gaussian 03, Revisión-B.03, Frisch, M. J.; Trucks, G. W.; Schlegel, H. B.; Scuseria, G. E.; Robb, M. A.; Cheeseman, J. R.; Montgomery, Jr., J. A.; Vreven, T.; Kudin, K. N.; Burant, J. C.; Millam, J. M.; Iyengar, S. S.; Tomasi, J.; Barone, V.; Mennucci, B.; Cossi, M.; Scalmani, G.; Rega, N.; Petersson, G. A.; Nakatsuji, H.; Hada, M.; Ehara, M., Toyota, K.; Fukuda, R.; Hasegawa, J.; Ishida, M.; Nakajima, T.; Honda, Y.; Kitao, O.; Nakai, H.; Klene, M.; Li, X.; Knox, J. E.; Hratchian, H. P.; Cross, J. B.; Adamo, C.; Jaramillo, J.; Gomperts, R.; Stratmann, R. E.; Yazyev, O.; Austin, A. J.; Cammi, R.; Pomelli, C.; Ochterski, J. W.; Ayala, P. Y.; Morokuma, K.; Voth, G. A.; Salvador, P.; Dannenberg, J. J.; Zakrzewski, V. G.; Dapprich, S.; Daniels, A. D.; Strain, M. C.; Farkas, O.; Malick, D. K.; Rabuck, A. D.; Raghavachari, K.; Foresman, J. B.; Ortiz, J. V.; Cui, Q.; Baboul, A. G.; Clifford, S.; Cioslowski, J.; Stefanov, B. B.; Liu, G.; Liashenko, A., Piskorz, P.; Komaromi, I.; Martin, R. L.; Fox, D. J.; Keith, T.; Al-Laham, M. A.; Peng, C. Y.; Nanayakkara, A.; Challacombe, M.; Gill, P. M. W.; Johnson, B.; Chen, W.; Wong, M. W.; Gonzalez, C.; Pople, J. A.; Pittsburgh, PA, 2003.

16. GaussView, Version 3.09, Dennington II, R.; Keith, T.; Millam, J.; Eppinnett, K.; Hovell, W. L.; Gilliland, R.; Semichem, Inc., Shawnee Mission, KS, 2003.

17. Roothaan, C. C. J.; Rev. Mod. Phys. 1951, 23, 69.

18. Day, G. M.; Trask, A.V.; Motherwell, W. D. S.; Jones, W.; Chem. Commun. 2006, 1, 54 .

19. Dodd, R. E.; Miller, R. E.; Wynne-Jones, W. F. K.; J. Chem. Soc. (Resumed) 1961, 2790.

20. Macoas, E. M. S.; Fausto, R.; Lundell, J.; Pettersson, M.; Khriachtchev, L.; Rasanen, M.; J. Phys. Chem. A 2001, 105, 3922.

21. Magee, J. L.; Shand Jr., W.; Eyring, H.; J. Am. Chem. Soc. 1941, 63, 677. 